\title{
3-D Analysis of Cortical Morphometry in Differential Diagnosis of Parkinson's Plus Syndromes: Mapping Frontal Lobe Cortical Atrophy in Progressive Supranuclear Palsy Patients
}

\author{
Duygu Tosun ${ }^{1}$, Simon Duchesne ${ }^{2}$, Yan Rolland ${ }^{3}$, Arthur W. Toga ${ }^{1}$, \\ Marc Vérin ${ }^{4}$, and Christian Barillot ${ }^{2}$ \\ ${ }^{1}$ Laboratory of Neuro Imaging UCLA School of Medicine, Los Angeles, CA, USA \\ ${ }^{2}$ Visages U746, INSERM-INRIA-CNRS-Univ-Rennes1, IRISA, Rennes, France \\ ${ }^{3}$ Dpartement de Radiologie et d'Imagerie Mdicale, CHR Hpital Sud, Rennes, France \\ ${ }^{4}$ Clinique Neurologique, Unit de Recherche Universitaire "Comportement et Noyaux \\ Gris Centraux", CHU de Rennes, Rennes, France
}

\begin{abstract}
With the ability to study brain anatomy in vivo using magnetic resonance imaging, studies on regional brain atrophy suggest possible improvements for differential diagnosis of movement disorders with parkinsonian symptoms. In this study, we investigate effects of different parkinsonian syndromes on the cortical gray matter thickness and the geometric shape of the cerebral cortex. The study consists of a total of 24 patients with a diagnosis of probable progressive supranuclear palsy (PSP), multiple systems atrophy (MSA) or idiopathic Parkinson's disease (IPD). We examine dense estimates of cortical gray matter thickness, sulcal depth, and measures of the curvature in a surface-based cortical morphometry analysis framework. Group difference results indicate higher cortical atrophy rate in the frontal lobe in PSP patients when compared to either MSA or IPD. These findings are indicative of the potential use of routine MRI and cortical morphometry in performing differential diagnosis in PSP, MSA and IPD.
\end{abstract}

Keywords: Cortical morphometry; Differential diagnosis; Parkinsonian syndromes; Sulcal atrophy.

\section{Introduction}

Neurodegenerative brain diseases possess unique morphological signatures; detection of such signs may prove useful in improving diagnosis, particularly for diseases in which there are few other diagnostic tools. Differential diagnosis of patients with Parkinsonian syndromes is a challenging but clinically important task, necessary to guide prognosis and treatment strategies. For instance, studies based on volumetric morphometry or visual atrophy ratings on MR images have 
shown differences in the regional rates of atrophy in the brainstem (midbrain and pons), the striatum, the cerebellum, the lateral and third ventricles as well as frontal and posterior inferior cortical regions 12. Midbrain atrophy is useful in differentiating progressive supranuclear palsy (PSP) from idiopathic Parkinson's disease (IPD) [3, while striatal abnormalities and cerebellar atrophy are more common in multiple-system atrophy (MSA) 4]. Even though these studies suggest that regional atrophy rates are potential morphological indexes required to establish an accurate diagnosis and follow disease progression, a rigorous differential diagnosis framework must account for a number of confounding factors.

First, regional atrophy rates are scalar measures that do not take into account the intrinsic geometry of the structures of interest. These are of particular concern when studying disease that have effects on the cerebral cortex because of its elaborate inward and outward folds in three dimensional space. Secondly, quantitative, rather than qualitative measurements must be performed for an objective assessment of atrophy.

In this study, we attempted to address these concerns by engineering an automated surface-based 3-D cortical morphometry framework that focuses on group differences in the geometry of the cerebral cortex. A battery of algorithms has been integrated in the proposed framework. These algorithms have been described and validated in detail in various publications [56778. We specifically aimed to test the hypothesis that there exists group differences in cortical atrophy patterns among PSP, MSA, and IPD. We tested this hypothesis by investigating the cortical gray matter thickness and the amount of dilation and filling of cortical sulcal spaces with cerebro-spinal fluid reflected on the cortical geometry.

\section{Materials and Methods}

\subsection{Subjects and Data Acquisition}

The study group consisted in 8 patients with a diagnosis of probable IPD without dementia ( 4 females and 4 males; age (sd) 58.5 (9.0)), 8 patients with a diagnosis of probable MSA (3 females and 5 males; age (sd) 58.6 (7.1)), and 8 patients with a diagnosis of probable PSP ( 4 females and 4 males; age (sd) 65.1 (9.4)). All groups were age-matched (Tukey-Kramer HSD, alpha = 0.05). All patients were seen at the Movement Disorder clinic of the Centre Hospitalier Universitaire de Rennes (Rennes, FR). Diagnosis was established following the NNIPPS (Neuroprotection and natural history and biology in parkinsonian plus syndromes 1) study clinical and imaging protocol, which include clinical criteria

\footnotetext{
1 The NNIPPS project is investigating the neuroprotective efficacy and safety of riluzole in MSA and PSP in the setting of a large, multi-center (49 sites), randomized, parallel-group, placebo-controlled trial in several European countries (France, UK, Germany). The imaging component of the NNIPPS project sought to understand structural MRI changes in these diseases, to define and validate a standardized acquisition protocol, and to construct prospectively validated image assessment instruments on MRI.
} 
from Gilman et al. 9] for MSA and from Litvan et al. [10] for PSP. The diagnosis was also supported by long-term (>36 months) clinical and neurological follow-up, and response to treatment in the case of IPD patients.

MR brain images of each participants were acquired on a GE Signa 1.5 Tesla MR scanner using a T1-weighted spoiled gradient echo (SPGR) pulse sequence with the following parameters: $\mathrm{TE}=5 \mathrm{~ms}$; $\mathrm{TR}=24 \mathrm{~ms} ; 45^{\circ}$ flip angle; matrix size $=256 \times 256 ; \mathrm{FOV}=240 \mathrm{~mm} \times 240 \mathrm{~mm}$; slice thickness $=1.2 \mathrm{~mm}$, with 124 contiguous sagittal cross-sections.

\subsection{Cortical Surface Reconstruction}

The skull, scalp, extra-cranial tissue, cerebellum, and brain stem (at the level of the diencephalon) were removed from each image data using a template-based segmentation with competitive level sets and fuzzy controls [8]. The Colin27 average brain from Montreal Neurological Institute database (the average of $27 \mathrm{~T} 1$ weighted MRI acquisitions from a single subject) served as the high definition structural brain template to define our anatomical target (i.e., cerebrum).The software yielded good cerebrum extraction in most cases; in several cases, however, additional manual editing was required to remove retained non-cerebral tissue. Cross-sectional view of T1-weighted MR image and the extracted cerebral volume are shown in Figs. 1(a) and (b).

After correcting for intensity non-uniformity in MR data using the Nonparametric Non-uniform intensity Normalization (N3) [11, each individual's cortical surface was extracted using a cortical reconstruction method using implicit surface evolution (CRUISE) technique developed by Han et al. [5] and shown to yield an accurate and topologically correct representation that lies at the geometric center of the cortical gray matter tissue [6]. CRUISE is a data-driven method combining a robust fuzzy segmentation method, an efficient topology correction algorithm, and a geometric deformable surface model. Each resulting cortical surface was represented as a triangle mesh comprising of approximately 300,000 mesh nodes. Reconstructed cortical surface for a sample brain is shown in Fig. 1(c).

\subsection{Cortical Morphometry}

Geometrically, the cerebral cortex is a thin, folded sheet of gray matter that is 1$5 \mathrm{~mm}$ thick, with an average thickness of approximately $2.5 \mathrm{~mm}$ [1213]. Cerebral degeneration can result in dilation of cortical sulcal openings and compensatory filling of freed space by cerebrospinal fluid (CSF), affecting the 3-D geometry of the cerebral cortex. This phenomenon is thought to be the combined result of the reduction of thickness of the gyral grey matter (GM) mantle and atrophy of gyral white matter (WM). We consider four measurements: cortical gray matter thickness to examine the cortical gray matter tissue atrophy; and geodesic sulcal depth along with two measures of curvature characteristics of the cortex to examine sulcal dilation. While cortical thickness is a metric of three-dimensional geometry of gray matter tissue sheet, geodesic sulcal depth and curvature measures rely on an overall two-dimensional approximation to this three-dimensional 


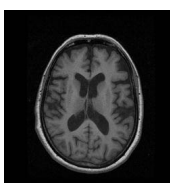

(a)

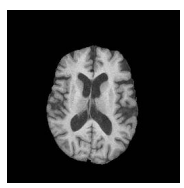

(b)

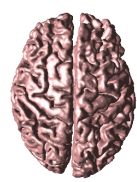

(c)

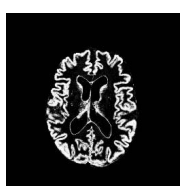

(d)

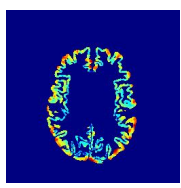

(e)

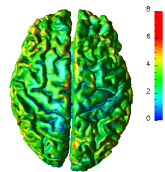

(f)

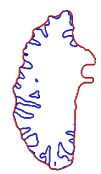

(g)

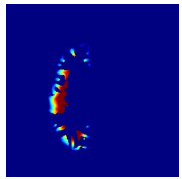

(h)

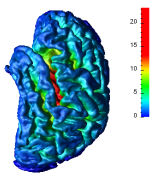

(i)

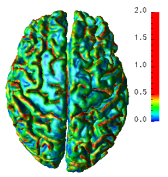

(j)

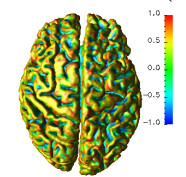

(k)

Fig. 1. Cross-sectional view of (a) T1-weighted MR image and (b) extracted cerebral volume; (c) Reconstructed cortical surface; Cross-sectional view of (d) gray matter tissue classification and (e) cortical gray matter thickness volume; (f) Cortical thickness displayed on the cortical surface; Cross-sectional view of (g) outer cortical surface and (h) geodesic sulcal depth volume for left hemisphere; (i) Sulcal depth displayed on the cortical surface; Curvature features $(\mathrm{j})$ curvedness and $(\mathrm{k})$ shape index displayed on the cortical surface

cortical sheet (i.e., central cortical surface [cf Section 2.2]). These measures are depicted in Figs. 1(d)-(k) and explained in detail herein.

Cortical Gray Matter Thickness. The cortical GM is bounded by the CSF on the outside, and by the WM on the inside. Adopting an Eulerian approach, the cortical thickness at each point in the GM tissue sheet can be defined to be the sum of the geodesic distances from the point to the GM/WM and GM/CSF interfaces. Let $\Omega \subset \Re^{3}$ (i.e., GM tissue sheet) be a spatial region with simply connected inner boundary $\partial_{0} \Omega$ (i.e., GM/WM interface) and outer boundary $\partial_{1} \Omega$ (i.e., GM/CSF interface). Then, the geodesic distances from each point $\boldsymbol{x} \in \Omega$ to the boundaries are computed by solving the following pair of PDEs:

$$
\begin{aligned}
& <\Delta D_{0}(\boldsymbol{x}), \boldsymbol{C}_{\mathbf{0}}(\boldsymbol{x})>=1, \text { with } D_{0}\left(\partial_{0} \Omega\right)=0, \\
& <\Delta D_{1}(\boldsymbol{x}), \boldsymbol{C}_{\mathbf{1}}(\boldsymbol{x})>=1, \text { with } D_{1}\left(\partial_{1} \Omega\right)=0 .
\end{aligned}
$$

According to these PDEs constructed from the geometry of the problem, $D_{0}(\boldsymbol{x})$ (and $D_{1}(\boldsymbol{x})$ ) is defined as the length of the correspondence trajectory $\boldsymbol{C}_{\mathbf{0}}$ (and $\boldsymbol{C}_{\mathbf{1}}$ ) that travel from $\boldsymbol{x}$ through $\Omega$ up to the $\partial_{0} \Omega$ (and $\partial_{1} \Omega$ ). Accordingly, the thickness $T(\boldsymbol{x})$ of $\Omega$ at $\boldsymbol{x}$ is

$$
T(\boldsymbol{x})=D_{0}(\boldsymbol{x})+D_{1}(\boldsymbol{x}) .
$$

The boundaries, $\partial_{0} \Omega$ and $\partial_{1} \Omega$, have sub-voxel resolution - i.e., partial voluming - and are usually represented as level sets of scalar functions. We use a Lagrangian approach to compute the values $D_{0}$ and $\boldsymbol{C}_{\mathbf{0}}$ at grid points in $\Omega$ adjacent to the boundary $\partial_{0} \Omega$. At the remain grid points in $\Omega$, we adopted a fast marching framework to compute the values $D_{0}$ and $\boldsymbol{C}_{\mathbf{0}}$ simultaneously [14. The values 
of $D_{1}$ and $\boldsymbol{C}_{\mathbf{1}}$ were computed in a similar way. This hybrid Eulerian-Lagrangian framework yields cortical thickness estimates with sub-voxel accuracy of a Lagrangian approach with the speed of an Eulerian approach. Cross-sectional view of the GM tissue classification and the corresponding cortical thickness volume of a sample subject and the resulting cortical thickness map on the surface are shown in Figs. 1(d)-(f).

Geodesic Sulcal Depth. Depth within sulcal regions is defined as the length of the geodesic path connecting any point on the cortical surface through the sulcal opening to the outer cortical surface (see Fig. 1(g)). Changes in sulcal depth are associated with a combination effect of non-uniform rate of GM thickness reduction across the cerebrum and atrophy of the gyral WM.

To compute the geodesic sulcal depth, we first generated an outer cortical surface that tightly surrounds the cortical surface without entering into the sulcal folds. The left and right cortical hemispheres were automatically identified by defining a cut around the corpus callosum using the knowledge of the locations of the midsagittal plan after a rigid alignment to the template brain's cortical surface. Focusing on one cortical hemisphere - i.e., allows to study sulcal regions in medical surface — , the outer cortical surface was computed by deforming the cortical surface according to an evolution equation:

$$
\Phi_{t}(\boldsymbol{x}, t)=R(\boldsymbol{x}) \kappa(\boldsymbol{x})\|\Delta \Phi(\boldsymbol{x}, t)\| .
$$

In this context, the deforming surface is implicitly embedded as the zero-level set of a scalar function $\Phi(\boldsymbol{x}, t)$ (i.e., signed distance function). $\Phi_{t}(\boldsymbol{x}, t)$ and $\Delta \Phi(\boldsymbol{x}, t)$ are the time derivative and the spatial gradient of $\Phi(\boldsymbol{x}, t)$, respectively. $\kappa(\boldsymbol{x})$, mean curvature function, and $R(\boldsymbol{x})$, barrier force, form the speed function that control the evolution of the level set function. While mean curvature component of the speed function aims to unfold sulcal folds, its barrier force term anchors the gyral crown points (i.e., visible cortex from outside) by preventing the surface from evolving in the inward direction. The result of this procedure is illustrated in Fig1(g) where a cross-section of the outer cortical surface (in red) is shown along with the original cortical surface (in blue).

The geodesic sulcal depth from the original cortical surface to the outer cortical surface can be calculated by the approach used for cortical thickness computation. We set $\partial_{0} \Omega$ and $\partial_{1} \Omega$ at the original cortical surface and at the outer surface, respectively; and $\Omega \subset \Re^{3}$ was defined as the spatial region between these boundary surfaces (i.e., sulcal openings). A cross section from the resulting sulcal depth volume of left cortical hemisphere of a sample subject and the corresponding surface map are shown in Figs. 1(h) and (i).

Curvature Characteristics of the Cortex. Enlargement of the cerebral sulci (i.e., dilation of cortical sulcal openings and compensatory filling of freed space by CSF) have a direct effect on the 3-D folding geometry of the cerebral cortex. An analysis on the curvature characteristics of the cortex assesses changes in the type and magnitude of cortical folds. We consider two curvature measures: 
shape index and curvedness. Given the two principal curvatures $\kappa_{1}$ and $\kappa_{2}$, where $\kappa_{1} \leq \kappa_{2}$, shape index $(\mathrm{SI})$ and curvedness $(\mathrm{CN})$ are defined as [15]

$$
\mathrm{SI}=\frac{2}{\pi} \arctan \frac{\kappa_{2}+\kappa_{1}}{\kappa_{2}-\kappa_{1}}, \quad \mathrm{CN}=\sqrt{\frac{\kappa_{1}^{2}+\kappa_{2}^{2}}{2}} .
$$

The scaling on the shape index is such that it provides a space of shapes with the topology of a line segment, i.e., $[-1,1]$. In this shape space, each surface point is classified as spherical cup point (i.e., $S I=-1$ ), saddle point (i.e., $S I=0$ ), spherical cap point (i.e., $S I=1$ ), or smooth transitions between these shape. This shape classification is invariant under global scaling of the surface. The missing scale (or size) information is captured by the curvedness measure. SI and $\mathrm{CN}$ maps of a sample individual are shown in Figs. 11(j) and (k), respectively. The SI successfully distinguished the cortical features such as sulci and gyri and CN gave the size of the folding.

\subsection{Cortical Normalization}

We used a surface-based 3-D cortical warping technique [7] to establish a dense point correspondence between anatomically homologous surface points of individual surfaces on a common reference surface coordinate system. This common coordinate system is defined by the template brain's cortical surface. The pairwised surface warping algorithm aims to align the geometric features of two cortical surfaces in a multi-scale framework using the sphere as a canonical joint coordinate system. Without requiring any manually identified landmark curves, this approach defines an anatomical homology between two cortical surfaces based on a similarity between the geometric features densely defined at each cortical surface point. Therefore, each subject cortical surface was spatially normalized with respect to the geometry of a representative template. Population averages as well as point-based statistical comparison were then computed within the common cortical coordinate system of the template brain.

\subsection{Statistical Analysis of the Cortical Shape Measures}

The cortical shape measures (i.e., cortical thickness, geodesic sulcal depth, shape index, and curvedness) were computed for 24 individuals in the study group. The shape measures were smoothed using a surface-based intrinsic isotropic smoothing filter of radius $10 \mathrm{~mm}$. The smoothing was performed to reduce computational noise on the shape measures.

Statistical analysis involved applying a hypothesis testing in order to determine whether 3 -D shape changes in cerebral cortex could differentiate Parkinson's plus syndromes MSA and PSP from IPD. We tested the equality of group means for each shape measure using Student's $t$-test. The significance level of each individual test was corrected for multiple comparisons using False Discovery Rate (FDR). 


\section{Results and Discussion}

The mean difference maps on the template brain surface are shown in the first three columns of Fig. 2, and the regions with significant mean difference $(p<0.05$ after FDR correction) are highlighted in the last three columns of Fig 2. Population average maps revealed different profiles of cortical thickness, sulcal depth, and curvedness across groups. Subtle yet significant differences in cortical folding shape (i.e. shape index) were found particularly in the frontal lobe when comparing PSP patients to IPD patients. GM thickness maps displayed tissue loss in frontal lobe and motor cortex, reaching significance in the motor cortex region when comparing PSP to MSA patients. When comparing MSA and PSP to IPD separately for differences in sulcal depth and curvedness maps, we observed reversed patterns of significance. These were more pronounced in MSA versus PSP significance maps, especially in the left frontal lobe area. Although our statistics on cortical thickness did not reveal a strongly distinguishing cortical atrophy pattern between MSA, PSP, and IPD groups, our strong findings on curvedness and sulcal depth measures in the frontal lobe suggest that atrophy of gyral WM in PSP patients is significantly different than MSA as well as IPD patients.

(a)
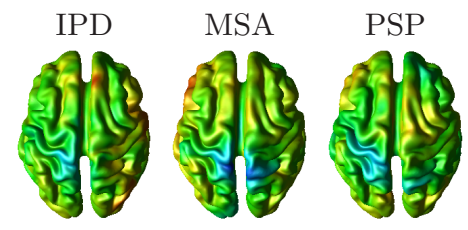

IPD vs MSA IPD vs PSP MSA vs PSP

(b)
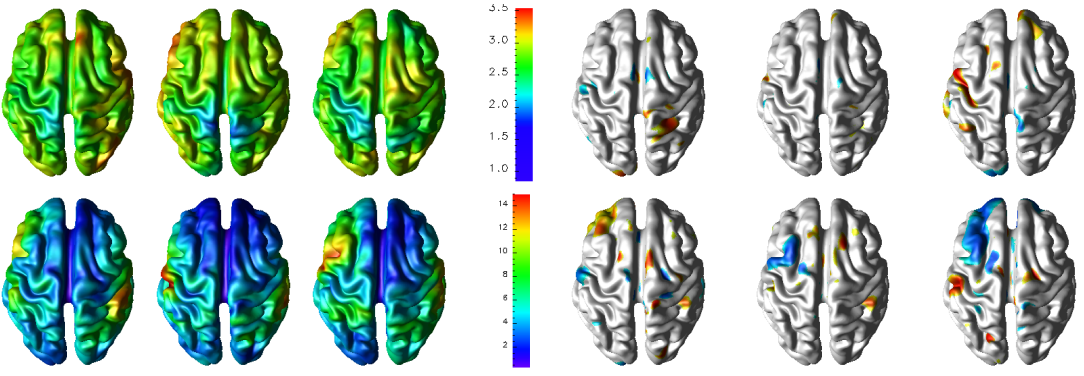

(c)
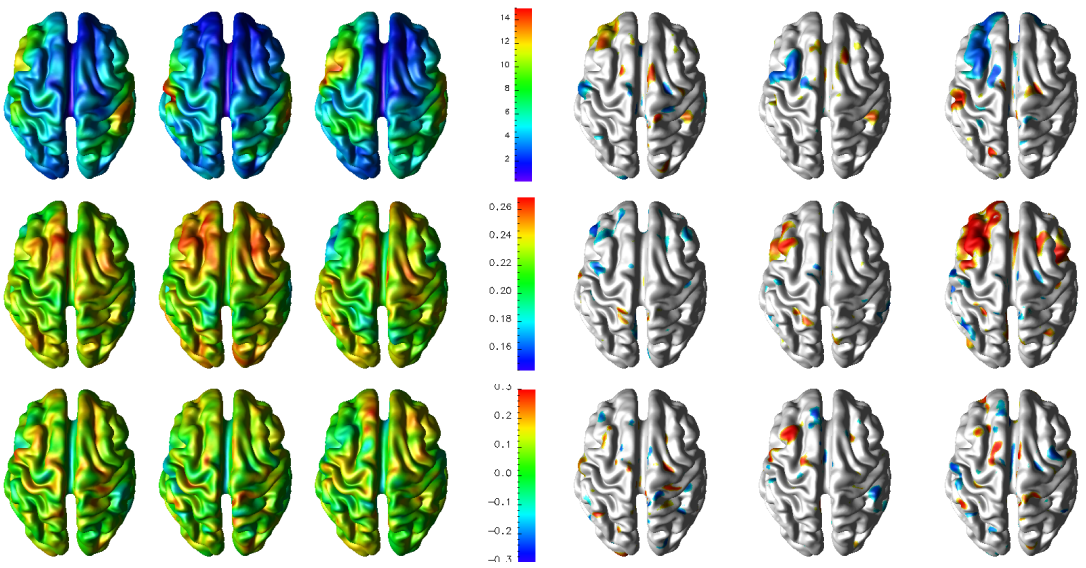

Population average maps

Significant mean difference maps

Fig. 2. Statistical analysis on (a) cortical thickness, (b) sulcal depth, (c) curvedness, and $(d)$ shape index measures. Color coding for significance maps: Population $1>$ Population 2 colored in red/yellow and Population $2>$ Population 1 colored in blue/cyan. $(p<0.05$, corrected for multiple comparisons using False Discovery Rate(FDR)). 
We interpret these finding as indicative of increased sulcal atrophy in the frontal lobe in PSP patients when compared to either MSA or IPD. Our findings in Broadmann areas 4 and 6 (i.e., the primary motor cortex and primary visual cortex areas), and Broadmann area 8 (i.e., frontal cortex area including the frontal eye field) agree with the cognitive decline in PSP patients that may, in part, be explained by the associated visual-motor deficit and frontal-lobe dysfunction [16. These preliminary results confirm our hypothesis of distinct patterns of sulcal atrophy and GM tissue loss, demonstrating the potential use of routine MRI and cortical morphometry in performing differential diagnosis in PSP, MSA and IPD. These findings encourage further investigation in a larger data set in the development of a differential diagnosis framework. Our morphometry analysis benefits from the use of different shape measures in studying cortical atrophy. Future work includes systematic studies on how different shape measures correlate in measuring change in 3-D cortical geometry and longitudinal evaluation of cerebral morphological changes.

\section{References}

1. Paviour, D.C., Price, S.L., Jahanshahi, M., Lees, A.J., Fox, N.C.: Longitudinal MRI in progressive supranuclear palsy and multiple system atrophy: rates and regions of atrophy. Brain 129(4), 1040-1049 (2006)

2. Oba, H., Yagishita, A., Terada, H., Barkovich, A.J., Kutomi, K., Yamauchi, T., Furui, S., Shimizu, T., Uchigata, M., Matsumura, K., Sonoo, M., Sakai, M., Takada, K., Harasawa, A., Takeshita, K., Kohtake, H., Tanaka, H., Suzuki, S.: New and reliable MRI diagnosis for progressive supranuclear palsy. Neurology 64(12)

3. Warmuth-Metz, M., Naumann, M., Csoti, I., Solymosi, L.: Measurement of the midbrain diameter on routine magnetic resonance imaging: a simple and accurate method of differentiating between parkinson disease and progressive supranuclear palsy. Arch. Neurol. 58, 1076-1079 (2001)

4. Bhattacharya, K., Saadia, D., Eisenkraft, B.e.a.: Brain magnetic resonance imaging in multiple-system atrophy and parkinson disease: a diagnostic algorithm. Arch. Neurol. 59, 835-842 (2002)

5. Han, X., Pham, L.D., Tosun, D., Rettmann, M.E., Xu, C., Prince, J.L.: CRUISE: Cortical reconstruction using implicit surface evolution. Neuro Image 23(3) (2004)

6. Tosun, D., Rettmann, M.E., Resnick, S.M., Pham, D.L., Prince, J.L.: Cortical reconstruction using implicit surface evolution: Accuracy and precision analysis. Neuro Image (2005)

7. Tosun, D., Prince, J.L.: Cortical surface alignment using geometry driven multispectral optical flow. In: Christensen, G.E., Sonka, M. (eds.) IPMI 2005. LNCS, vol. 3565, Springer, Heidelberg (2005)

8. Ciofolo, C., Barillot, C.: Brain segmentation with competitive level sets and fuzzy control. In: Christensen, G.E., Sonka, M. (eds.) IPMI 2005. LNCS, vol. 3565, pp. 10-15. Springer, Heidelberg (2005)

9. Gilman, S., Low, P.A., Quinn, N., Albanese, A., Ben-Shlomo, Y., Fowler, C.J., Kaufmann, H., Klockgether, T., Lang, A.E., Lantos, P.L., Litvan, I., Mathias, C., Oliver, E., Robertson, D., Schatz, I., Wenning, G.K.: Consensus statement on the diagnosis of multiple system atrophy. J. Neurol. Sci. 163, 94-98 (1999) 
10. Litvan, I., Agid, Y., Calne, D., Campbell, G., Dubois, B., Duvoisin, R.C., Goetz, C.G., Golbe, L.I., Grafman, J., Growdon, J.H., Hallett, M., Jankovic, J., Quinn, N.P., Tolosa, E., Zee, D.S.: Clinical research criteria for the diagnosis of progressive supranuclear palsy (steele-richardson-olszewski syndrome): report of the ninds-spsp international workshop. Neurology 47, 1-9 (1996)

11. Sled, J.G., Zijdenbos, A.P., Evans, A.C.: A non-parametric method for automatic correction of intensity non-uniformity in mri data. IEEE Trans. Medical Imaging 17, 87-97 (1998)

12. Griffin, L.D.: The intrinsic geometry of the cerebral cortex. J. Theoretical Biology 166(3), 261-273 (1994)

13. Beatty, J.: The Human Brain: Essentials of Behavioral Neuroscience. Sage Publications, Inc, Thousand Oaks (2001)

14. Prados, E., Soatto, S.: Fast marching method for generic shape from shading. In: Paragios, N., Faugeras, O., Chan, T., Schnörr, C. (eds.) VLSM 2005. LNCS, vol. 3752, Springer, Heidelberg (2005)

15. Koenderink, J.J., van Doorn, A.J.: Surface shape and curvature scales. Image and Vision Computing 10(8), 557-565 (1992)

16. Pillon, B., Dubois, B., Lhermitte, F., Agid, Y.: Heterogeneity of cognitive impairment in progressive supranuclear palsy, Parkinson's disease, and Alzheimer's disease. Neurology 36(9), 1179 (1986) 